\title{
A CONSTRUCTION OF MODULAR REPRESENTATIONS OF CLASSICAL LIE ALGEBRAS
}

\author{
KARL M. PETERS AND ZHIYONG SHI
}

(Communicated by Eric Friedlander)

\begin{abstract}
In this paper, we construct and analyze new classes of modular representations of classical Lie algebras of type $C$ and type $A$. These representations include a class of pointed torsion free representations, a class of irreducible nonrestricted representations, and a class of indecomposable representations of arbitrary high dimension. The construction is based on the realization of these Lie algebras in the modular Weyl algebras acting on truncated polynomial algebras. We also classify all the irreducible representations of the modular Weyl algebra.
\end{abstract}

\section{INTRODUCTION}

Let $k$ be an algebraically closed field of positive characteristic $p>3, k_{p}$ the prime field, and $\mathfrak{g}$ any restricted Lie algebra. For any element $x \in \mathfrak{g}$, the element $x^{p}-x^{[p]}$ belongs to the center of the enveloping algebra $\mathfrak{U}(\mathfrak{g})$ of $\mathfrak{g}$. As a consequence, it is possible to index the irreducible representations of $\mathfrak{g}$ by algebra homomorphisms $\mathscr{O}=\left\langle x^{p}-x^{[p]} \mid x \in \mathfrak{g}\right\rangle \longrightarrow k$. Such characters may also be considered as linear mappings in $\mathfrak{g}^{*}$. A representation $\rho$ of $\mathfrak{g}$ is said to admit a character $\chi \in \mathfrak{g}^{*}$ if

$$
\rho(x)^{p}-\rho\left(x^{[p]}\right)=\chi(x)^{p} \rho(1)
$$

for all elements $x \in \mathfrak{g}$. A representation is restricted if it admits the character $\chi=0$ and nonrestricted if $\chi \neq 0$. Let $\mathfrak{U}(\mathfrak{g}, \chi)$ be the $\chi$-enveloping algebra, the finite-dimensional, associative algebra with 1 whose representations are equivalent to the representations of $\mathfrak{g}$ admitting $\chi$. We focus our attention on the representations of the restricted, classical Lie algebras. Henceforth, $\mathfrak{g}$ denotes a semisimple classical Lie algebra over $k$.

The representations of classical $\mathfrak{g}$ are closely related to the representations of any semisimple, simply connected algebraic group $G$ with $\operatorname{Lie}(G)=\mathfrak{g}$. However, the differential of a representation of $G$ is a restricted representation of $\mathfrak{g}$. As a consequence, most standard examples of modular representations of $\mathfrak{g}$ are restricted. The importance of the nonrestricted representations to the classification problem of all irreducible representations of $\mathfrak{g}$ was recognized by Kac and Weisfeiler (see [WK] and [KW]). More recent developments can be found

Received by the editors January 27, 1993.

1991 Mathematics Subject Classification. Primary 17B10, 17B50. 
in [FP1-3], where Friedlander and Parshall study representations of $\mathfrak{U}(\mathfrak{g}, \chi)$. In this paper, we further examine the relevance of nonrestricted representations to general representation theory of classical Lie algebras $\mathfrak{g}$.

We employ the following notation. Let $h$ denote a fixed Cartan subalgebra of such a $\mathfrak{g}$. Let $\Phi=\Phi^{+} \cup \Phi^{-}$be the root system of $\mathfrak{g}$ relative to $\mathfrak{h}, \mathfrak{g}_{\gamma}$ the root space of $\mathfrak{g}$ corresponding to the root $\gamma$, and $\Delta=\left\{\alpha_{1}, \alpha_{2}, \ldots, \alpha_{\ell}\right\}$ the simple roots. For each $\gamma \in \Phi^{+}$, we fix $e_{\gamma} \in \mathfrak{g}_{\gamma}, h_{\gamma} \in \mathfrak{h}$, and $e_{-\gamma} \in \mathfrak{g}_{-\gamma}$ so that $\left\{e_{\gamma}, h_{\gamma}, e_{-\gamma} \mid \gamma \in \Phi^{+}\right\}$is a set of Chevalley generators of $\mathfrak{g}$. We abbreviate $e_{i}=e_{\alpha_{i}}, h_{i}=h_{\alpha_{i}}$, and $f_{i}=e_{-\alpha_{i}}$. The mapping $[p]: \mathfrak{g} \longrightarrow \mathfrak{g}$ defined by $e_{\gamma}^{[p]}=0$ and $h_{\gamma}^{[p]}=h_{\gamma}$ makes $\mathfrak{g}$ a restricted Lie algebra.

Nonrestricted modular representations of classical Lie algebras arise, by analogy, from torsion free representations in characteristic 0 . A representation of $\mathfrak{g}$ is torsion free if all the root vectors $e_{\gamma}$ act injectively. The theory of torsion free representations in characteristic 0 has been developed by Britten and Lemire [BL1, 2], Fernando [F], and Britten, Futorny, and Lemire [BFL]. Fernando proves that only $\mathfrak{g}$ of type $A_{\ell}$ or $C_{\ell}$ have torsion free representations, which are always infinite dimensional. In [BL1], the pointed torsion free representations of $\mathfrak{g}$ ("pointed" refers to the irreducibility and the existence of a one-dimensional weight space) are classified and are realized by the embedding of $\mathfrak{g}$ in the Weyl algebras over $\mathbb{C}$. Nonrestricted modular representations share many features with these infinite-dimensional, torsion free representations in characteristic 0 . For example, any representation admitting a character $\chi$ with $\chi\left(e_{\gamma}\right) \neq 0$ has $e_{\gamma}$ acting injectively since $e_{\gamma}^{p}$ acts as the scalar $\chi\left(e_{\gamma}\right)^{p}$.

In this paper, we consider connections of the above results with modular representation theory. In the next section, we begin by classifying all the irreducible representations of the modular Weyl algebra $\mathscr{W}_{\ell}$. Then we construct classes of representations of $\mathscr{W}_{\ell}$ parameterized by $2 \ell$-tuples of elements from the field. These representations are very natural, realized as collections of truncated polynomials, and include all the irreducibles. In the third section, representations of classical Lie algebras are obtained from this Weyl algebra construction by restricting to well-known Lie subalgebras of $\mathscr{W}_{\ell}$ of types $C$ and $A$. Depending on the parameters and the level of truncation, this construction yields irreducible nonrestricted representations, pointed torsion free representations, or indecomposable representations of arbitrary high dimension for these classical Lie algebras.

\section{Modular Weyl algebras}

Let $R$ be any associative algebra with 1 over $k$. We denote by Rep $R$ the set of all equivalence classes of finite-dimensional irreducible representations. Then by Schur's lemma, we have a canonical map (the central character map)

$$
\operatorname{Rep} R \stackrel{\phi}{\longrightarrow} \operatorname{Rep} Z(R),
$$

where $Z(R)$ is the center of $R$. By Nakayama's lemma, $R / R \operatorname{ker} \chi$ is a nonzero finite-dimensional $k$-algebra for any $\chi \in \operatorname{Rep} Z(R)$ and, hence, has a finitedimensional irreducible representation. This implies that the map $\phi$ in (2.1) is surjective. If $V \in \operatorname{Rep} R$, then $\chi=\phi(V)$ is called the (central) character of $V$. Note that we may replace $Z(R)$ by any central subalgebra $Z \subset Z(R)$.

Applying the preceding paragraph to the universal enveloping algebras $\mathfrak{U}(g)$ 
and the central subalgebra $\mathscr{O}$, the next result stands in contrast to the characteristic 0 results of Fernando.

Proposition 2.2. For any $\chi \in \mathfrak{g}^{*}$, there exists a (finite-dimensional) irreducible representation of $\mathfrak{g}$ admitting the character $\chi$. In particular, there exist irreducible torsion free representations for classical $\mathfrak{g}$ of any type.

The modular Weyl algebra over the field $k$ is defined by the same relations as in characteristic 0 , that is, $\mathscr{W}_{\ell}$ is the associative algebra with 1 generated by $\partial_{i}, x_{i}, 1 \leq i \leq \ell$ subject to relations

$$
\left[\partial_{i}, \partial_{j}\right]=\left[x_{i}, x_{j}\right]=0, \quad\left[\partial_{i}, x_{j}\right]=\delta_{i j} .
$$

It is easy to see that $\mathscr{W}_{\ell}$ is the tensor product of $\ell$ copies of $\mathscr{W}_{1}$. We study $\mathscr{W}:=\mathscr{W}_{1}$ first and for simplicity of notation suppress the subscripts for the generators $\partial_{1}$ and $x_{1}$.

By induction, we can prove the following formulae for $i \in \mathbb{Z}_{\geq 0}$ :

$$
\left[\partial^{i}, x\right]=i \partial^{i-1}, \quad\left[\partial, x^{i}\right]=i x^{i-1} .
$$

The proof of the next lemma follows from a standard argument using filtrations.

Lemma 2.4. The center $Z(\mathscr{W})$ of $\mathscr{W}$ is equal to the subalgebra generated by the $\partial^{p}$ and $x^{p}$. Moreover, the center is $k\left[\partial^{p}, x^{p}\right]$, which is isomorphic to the two-variable polynomial algebra over $k$.

So $\mathscr{W}$ is a finitely generated module over its center and has no infinitedimensional irreducible representations (see [SF, §5.2]). Since $\operatorname{Rep} Z=k \times k$, any irreducible representation of $\mathscr{W}$ uniquely determines a pair $(a, b) \in k \times k$. Indeed, we have

Proposition 2.5. The central character map of sets from $\operatorname{Rep} \mathscr{W} \stackrel{\phi}{\longrightarrow} k \times k$ is bijective and any irreducible representation of $\mathscr{W}$ is of dimension $p$.

Proof. Suppose $V$ is an irreducible representation of $\mathscr{W}$ with the central character $\chi$. Let $\chi\left(\partial^{p}\right)=a, \chi\left(x^{p}\right)=b$. Then $a^{1 / p}$ is an eigenvalue of $\partial$. Take any eigenvector $v \in V$ of $a^{1 / p}$. We claim that

$$
V=\bigoplus_{i=0}^{p-1} k x^{i} v .
$$

Let $V_{0}=\sum_{i=0}^{p-1} k x^{i} v$. By (2.3), we have

$$
\partial x^{i} v=a^{1 / p} x^{i} v+i x^{i-1} v \text { or }\left(\partial-a^{1 / p}\right) x^{i} v=i x^{i-1} v ;
$$

hence, $V_{0}$ is invariant under both $\partial$ and $x$ and $V_{0}=V$. Let $\sum_{i=0}^{m} c_{i} x^{i} v=0$ be an expression with $0 \leq m \leq p-1$ as small as possible such that $c_{m} \neq 0$. Applying $\left(\partial-a^{1 / p}\right)^{m}$ to this expression, we get $c_{m} m ! v=0$ by (2.7), which is impossible by the choice of $m$. This implies that $v, x v, \ldots, x^{p-1} v$ are linearly independent and $V$ is of dimension $p$. Since the actions of $\partial$ and $x$ on $V$ are uniquely determined by $a$ and $b, \phi$ must be injective and the result follows.

Corollary 2.8. (1) Any irreducible representation of $\mathscr{W}_{\ell}$ is of the form $\bigotimes_{i=1}^{\ell} V_{i}$, where $V_{i}$ is any irreducible representation of the subalgebra $\left\langle\partial_{i}, x_{i}\right\rangle \cong \mathscr{W}$. Any irreducible representation of $\mathscr{W}_{\ell}$ has the dimension $p^{\ell}$.

(2) The character map of $\operatorname{Rep} \mathscr{W}_{\ell} \rightarrow \operatorname{Rep} Z\left(W_{\ell}\right)=k^{2 \ell}$ is bijective. 
There is a natural representation of the modular Weyl algebra $\mathscr{W}_{\ell}$ on a polynomial ring with coefficients in $k$ where $\partial_{i}$ and $x_{i}$ act as the derivative $\partial / \partial x_{i}$ and multiplication by $x_{i}$, respectively. In fact, any irreducible module of $\mathscr{W}_{\ell}$ admitting any character may be realized in a similar manner by truncating the polynomials and manipulating this action. For $\ell$-tuples $\mathbf{b}=\left(b_{i}\right) \in(k)^{\ell}$ and $\mathbf{m}=\left(m_{i}\right) \in\left(\mathbb{Z}_{>0}\right)^{\ell}$ consider the truncation algebra

$$
\mathscr{T}(\mathbf{m}, \mathbf{b}):=k\left[x_{1}, \ldots, x_{\ell}\right] /\left(x_{1}^{\left(p^{m_{1}}\right)}-b_{1}, \ldots, x_{\ell}^{\left(p^{m_{\ell}}\right)}-b_{\ell}\right) .
$$

Let $e^{\mathbf{a}^{1 / p} \mathbf{x}}:=e^{\sum_{i=1}^{\ell} a_{i}^{1 / p} x_{i}}$ be the formal exponential defined so that $\partial / \partial x_{i} \cdot e^{\mathbf{a}^{1 / p} \mathbf{x}}=$ $a_{i}^{1 / p} e^{\mathbf{a}^{1 / p} \mathbf{x}}$. On the vector space $\mathscr{E}(\mathbf{m}, \mathbf{a}, \mathbf{b}):=e^{\mathbf{a}^{1 / p}} \mathbf{x} \mathscr{T}(\mathbf{m}, \mathbf{b})$, let $\partial_{i}$ act as the partial derivative $\partial / \partial x_{i}$ and $x_{i}$ act as multiplication by $x_{i}$. Using the formal product rule, it is straightforward to confirm that the defining relations of $\mathscr{W}_{\ell}$ are satisfied. Thus, we have defined a representation of $\mathscr{W}_{\ell}$ on $\mathscr{E}(\mathbf{m}, \mathbf{a}, \mathbf{b})$. Let $\mathbf{1}=(1,1, \ldots, 1)$.

Proposition 2.10. The representation of $\mathscr{W}_{\ell}$ on $\mathscr{E}(\mathbf{1}, \mathbf{a}, \mathbf{b})$ is irreducible and admits the arbitrarily chosen character $(\mathbf{a}, \mathbf{b}) \in k^{\ell} \times k^{\ell}$.

Proof. Since the representation has dimension $p^{\ell}$, it is irreducible by Proposition 2.5. The character may be calculated using the fact that $e^{\mathbf{a}^{1 / \mathbf{p}_{\mathbf{x}}}} 1$ is an eigenvector of $\partial_{i}$ with eigenvalue $a_{i}^{1 / p}$ and $x_{i}^{p} \cdot e^{\mathbf{a}^{1 / p} \mathbf{x}} 1=b_{i} e^{\mathbf{a}^{1 / p} \mathbf{x}} 1$.

It is possible to realize some of the irreducible $\mathscr{W}_{\ell}$ representations $\mathscr{E}(\mathbf{1}, \mathbf{a}, \mathbf{b})$ in another more useful manner. Let $k^{\times}$be the nonzero elements of $k$ and $\mathbf{b} \in\left(k^{\times}\right)^{\ell}$. For any $\ell$-tuple of nonnegative integers $\mathbf{n}$, let $x^{\mathbf{n}}$ be the monomial $x_{1}^{n_{1}} x_{2}^{n_{2}} \cdots x_{\ell}^{n_{\ell}}$ and $x^{\epsilon_{i}}=x_{i}$ in $\mathscr{T}(\mathbf{m}, \mathbf{b})$. We may define representations of $\mathscr{W}_{\ell}$ on the vector space $\mathscr{T}(\mathbf{m}, \mathbf{b})$ indexed by $\ell$-tuples $\mathbf{a}=\left(a_{i}\right) \in k^{\ell}$. Set

$$
x_{i} \cdot x^{\mathbf{n}}=x^{\mathbf{n}+\epsilon_{i}} \text { and } \partial_{i} \cdot x^{\mathbf{n}}=b_{i}^{-1}\left(n_{i}+a_{i}\right) x^{\mathbf{n}+\left(p^{m_{i}}-1\right) \epsilon_{i}} .
$$

The division by $b_{i}$ in the action of $\partial_{i}$, and thus the assumption that each $b_{i} \neq 0$, is needed to insure that $\left[\partial_{i}, x_{i}\right]$ acts as the identity. It follows that (2.11) defines a $\mathscr{W}_{\ell}$ representation which we denote by $\mathscr{P}=\mathscr{P}(\mathbf{m}, \mathbf{a}, \mathbf{b})$. One of the advantages of working with $\mathscr{P}$ over $\mathscr{E}$ is that monomials of the truncation algebra are mapped to monomials under the actions of both $\partial_{i}$ and $x_{i}$. The natural representation of $\mathscr{W}_{\ell}$ on $\mathscr{T}(\mathbf{m}, \mathbf{b})$ is recovered by setting $\mathbf{a}=(0,0, \ldots, 0)$.

We note that the representation $\mathscr{P}(\mathbf{1}, \mathbf{a}, \mathbf{b})$ of $\mathscr{W}_{\ell}$ has dimension $p^{\ell}$ and is thus irreducible. It admits the character $\chi$ with $\chi\left(\partial_{i}^{p}\right)=\left(a_{i}^{p}-a_{i}\right) b_{i}^{-1}$ and $\chi\left(x_{i}^{p}\right)=b_{i}$ for all $i=1,2, \ldots, \ell$. It is clear that since $b_{i} \neq 0, x_{i}$ will act torsion freely. If $a_{i} \notin k_{p}, \partial_{i}$ acts torsion freely. It also follows that $\mathscr{P}(\mathbf{1}, \mathbf{a}, \mathbf{b})$ and $\mathscr{P}\left(\mathbf{1}, \mathbf{a}^{\prime}, \mathbf{b}^{\prime}\right)$ are equivalent $\mathscr{W}_{\ell}$-representations iff $\mathbf{b}=\mathbf{b}^{\prime}$ and $a_{i}-a_{i}^{\prime} \in k_{p}$ for all $i$.

\section{NONRESTRICTED REPRESENTATIONS}

As in characteristic 0 (see [BL2]), there exist natural embeddings of classical Lie algebras of types $C_{\ell}$ and $A_{\ell-1}$ in the Weyl algebra $\mathscr{W}_{\ell}$. We shall denote these Lie algebras contained in $\mathscr{W}_{\ell}$ by $\mathfrak{g}(C)$ and $\mathfrak{g}(A)$, respectively. In what follows, results on representations of $\mathfrak{g}(C)$ will lead to analogous results on rep- 
resentations of $\mathfrak{g}(A)$. Furthermore, when working with $\mathfrak{g}(A)$ representations, we will now assume that $p$ does not divide $\ell$. It is necessary to have a precise description of the embedding of $\mathfrak{g}(C)$ in $\mathscr{W}_{\ell}$.

Proposition 3.1. The vector subspace $\mathfrak{g}(C)$ of $\mathscr{W}_{\ell}$ defined by

$$
\mathfrak{g}(C)=\sum_{i, j=1}^{\ell}\left\langle\frac{1}{2}\left(\partial_{i} x_{j}+x_{j} \partial_{i}\right)\right\rangle+\left\langle x_{i} x_{j}\right\rangle+\left\langle\partial_{i} \partial_{j}\right\rangle
$$

is a Lie subalgebra of $\mathscr{W}_{\ell}$ of type $C_{\ell}$. Furthermore, the following elements of $\mathscr{W}_{\ell}$ satisfy the Serre relations (see [B]) corresponding to a type $C_{\ell}$ Cartan matrix.

$$
\begin{array}{llll}
h_{i} \mapsto x_{i} \partial_{i}-x_{i+1} \partial_{i+1}, & e_{i} \mapsto x_{i} \partial_{i+1}, & f_{i} \mapsto x_{i+1} \partial_{i}, & \\
h_{\ell} \mapsto x_{\ell} \partial_{\ell}+\frac{1}{2}, & e_{\ell} \mapsto \frac{1}{2} x_{\ell}^{2}, & f_{\ell} \mapsto-\frac{1}{2} \partial_{\ell}^{2}, & 1 \leq i \leq \ell-1,
\end{array}
$$

and, relative to the Cartan subalgebra $\mathfrak{h}=\left\langle h_{1}, h_{2}, \ldots, h_{\ell}\right\rangle$,

$$
\left\{x_{i} \partial_{j} \mid 1 \leq i<j \leq \ell\right\} \cup\left\{x_{i} x_{j} \mid 1 \leq i \leq j \leq \ell\right\}
$$

is a set of positive root vectors of $\mathfrak{g}(C)$ and

$$
\left\{x_{i} \partial_{j} \mid 1 \leq j<i \leq \ell\right\} \cup\left\{\partial_{i} \partial_{j} \mid 1 \leq i \leq j \leq \ell\right\}
$$

is a set of negative root vectors for $\mathfrak{g}(C)$.

Proof. Using the assumption that $p \neq 2$, the first part of the theorem is proved in the same manner as in characteristic 0 [D, Lemma 4.6.9]. The remaining statements concerning the Serre generators and root vectors can be confirmed by calculations using the defining relations of $\mathscr{W}_{\ell}$.

Corollary 3.2. The elements $e_{i}, h_{i}, f_{i}$ for $i=1,2, \ldots, \ell-1$ of Proposition 3.1 generate a Lie algebra $\mathfrak{g}(A)$ of type $A_{\ell-1}$ in $\mathscr{W}_{\ell}$. The set

$$
\left\{x_{i} \partial_{j} \mid 1 \leq i \neq j \leq \ell\right\}
$$

is a set of root vectors of $\mathfrak{g}(A)$ relative to the Cartan subalgebra $\hat{\mathfrak{h}}=$ $\left\langle h_{1}, h_{2}, \ldots, h_{\ell-1}\right\rangle$.

A most interesting class of representations is constructed by restricting the $\mathscr{W}_{\ell}$ representation $\mathscr{P}=\mathscr{P}(\mathbf{m}, \mathbf{a}, \mathbf{b})$ to the Lie subalgebras $\mathfrak{g}(C)$ and $\mathfrak{g}(A)$. Let $|\mathbf{n}|=\sum_{i=1}^{\ell} n_{i}$ and $\hat{m}=\min \left(m_{1}, m_{2}, \ldots, m_{\ell}\right)$. Two monomials $x^{\mathbf{n}}$ and $x^{\mathbf{n}}$ of $\mathscr{P}$ are said to have the same degree if $|\mathbf{n}| \equiv|\overline{\mathbf{n}}|$ modulo $p^{\hat{m}}$. By examining the basis elements of $\mathfrak{g}(A)$ given in Corollary 3.2, we see that the action of $\mathfrak{g}(A)$ on $\mathscr{P}$ is degree preserving. In fact,

$$
\mathscr{P}=\bigoplus_{j=0}^{p^{\dot{m}}-1} \mathscr{P}_{j}
$$

as $\mathfrak{g}(A)$ modules where we define $\mathscr{P}_{j}$ to be the vector subspace of $\mathscr{P}$ with basis consisting of all monomials with degree $j$. We analyze the structure of the $\mathfrak{g}(C)$ representations $\mathscr{P}$ and the $\mathfrak{g}(A)$ representations $\mathscr{P}_{j}$.

Under certain conditions on the scalars a, the representations of this construction are torsion free. 
Theorem 3.3. If $a_{i} \notin k_{p}$ for all $i=1,2, \ldots, \ell$, then $\mathscr{P}(\mathbf{m}, \mathbf{a}, \mathbf{b})$ is a torsion free representation of both $\mathfrak{g}(C)$ and $\mathfrak{g}(A)$.

Proof. Let $\tilde{a}_{i}$ denote $a_{i}^{p}-a_{i}$. We have:

$$
\begin{aligned}
& \left(x_{i} \partial_{j}\right)^{\left(p^{m_{i}+m_{j}}\right)} \text { acts as } b_{i}^{\left(p^{m_{j}}\right)} b_{j}^{\left(-p^{m_{i}}\right)} \tilde{a}_{j}^{\left(p^{m_{i}+m_{j}-1}\right)} \text { for } 1 \leq i \neq j \leq \ell, \\
& \left(x_{i} x_{j}\right)^{\left(p^{m_{i}+m_{j}}\right)} \text { acts as } b_{i}^{\left(p^{m_{j}}\right)} b_{j}^{\left(p^{m_{i}}\right)} \text { for } 1 \leq i \leq j \leq \ell, \\
& \left(\partial_{i} \partial_{j}\right)^{\left(p^{m_{i}+m_{j}}\right)} \text { acts as } b_{i}^{\left(-p^{m_{j}}\right)} \tilde{a}_{i}^{\left(p^{m_{i}+m_{j}-1}\right)} b_{j}^{\left(-p^{m_{i}}\right)} \tilde{a}_{j}^{\left(p^{m_{i}+m_{j}-1}\right)} \text { for } 1 \leq i \leq j \leq \ell .
\end{aligned}
$$

Each of these scalars is nonzero by assumption. Thus, all root vectors of $\mathfrak{g}(C)$ and $\mathfrak{g}(A)$ (see Proposition 3.1 and Corollary 3.2) act injectively on $\mathscr{P}$.

The focus on the classical Lie algebra $\mathfrak{g}(C)$ (and $\mathfrak{g}(A)$ ) is partly due to the fact that any monomial $x^{\mathbf{n}}$ is a weight vector relative to $\mathfrak{h}$ (to $\hat{\mathfrak{h}}$ ) of weight $\lambda_{\mathbf{n}}$ where we define

$$
\lambda_{\mathbf{n}}\left(h_{i}\right)=n_{i}+a_{i}-n_{i+1}-a_{i+1} \text { and } \lambda_{\mathbf{n}}\left(h_{\ell}\right)=n_{i}+a_{n}+\frac{1}{2} .
$$

From these formulas and the fact that the truncated monomials form a basis of $\mathscr{P}$, it is clear that the $\mathfrak{g}(C)$ representation $\mathscr{P}$ is a weight representation with $p^{\ell}$ distinct weights and all weight spaces have the same dimension, $p^{|m|-\ell}$. Similarly, the $\mathfrak{g}(A)$ representation $\mathscr{P}_{j}$ has $p^{\ell-1}$ distinct weight spaces each of dimension $p^{|\mathrm{m}|-(\ell-1)-\hat{m}}$. Representations whose weight spaces all have the same dimension $d$ are said to be of degree $d$. Also, different weights of $\mathscr{P}$ $\left(\mathscr{P}_{j}\right)$ differ only by elements of the prime field $k_{p}$, and all such possible $p^{\ell}$ (respectively, $p^{\ell-1}$ ) functionals occur as weights of these representations. Representations of classical Lie algebras $\mathfrak{g}$ of fixed degree $d$ with $p^{\text {rank(g) }}$ distinct weights are frequently encountered in the modular theory.

The realization of $\mathfrak{g}(C)$ and $\mathfrak{g}(A)$ in $\mathscr{W}_{\ell}$ makes it possible to move between and within weight spaces of $\mathscr{P}$ and $\mathscr{P}_{j}$.

Lemma 3.5. (1) For any two monomials $x^{\mathbf{n}}$ and $x^{\overline{\mathbf{n}}}$ of $\mathscr{P}$, there is an element $\sigma_{\operatorname{nin}}$ of $\mathfrak{U}(\mathfrak{g}(C))$ that has the following properties:

(a) $\sigma_{\mathrm{na}}\left(x^{\mathbf{n}}\right)=x^{\mathbf{n}}$ and for any monomial $x^{\mathbf{r}}$ in the $\lambda_{\mathbf{n}}$ weight space, $\sigma_{\mathbf{n a n}}\left(x^{\mathbf{r}}\right)$ $=s_{\mathbf{r}} x^{\mathbf{r}}$ for some nonzero $s_{\mathbf{r}} \in k$ and monomial $x^{\overline{\mathbf{r}}}$ in the $\lambda_{\overline{\mathbf{n}}}$ weight space,

(b) $\sigma_{\text {nñ }}$ acts injectively,

(c) $\sigma_{\mathrm{nn}}^{\left(p^{|m|}\right)}$ acts as a nonzero scalar.

Furthermore, if $\lambda_{\mathrm{n}}=\lambda_{\overline{\mathbf{n}}}$, then $\sigma_{\mathrm{nn}}$ is an element of the center of $\mathfrak{U}(\mathfrak{g}(C))$.

(2) If $a_{t} \notin k_{p}$ for some $t=1,2, \ldots, \ell$, then for any two monomials $x^{\mathrm{n}}$ and $x^{\mathfrak{n}}$ of $\mathscr{P}_{j}$, there is an element $\sigma_{\mathrm{nn}}$ of $\mathfrak{U}(\mathfrak{g}(A))$ that has the same properties as above.

Proof. For $\mathfrak{g}(C)$, let $l_{i}$ be the unique integer between 0 and $p^{m_{i}}-1$ congruent to $\left(\bar{n}_{i}-n_{i}\right) / 2$ modulo $p^{m_{i}}$. Using the fact that $x_{i} x_{i}$ is a root vector of $\mathfrak{g}(C)$, there exists a scalar $s \in k$ (involving only the $b_{i}$ ) such that

$$
\sigma_{\mathrm{nn}}=1 / s\left(x_{1} x_{1}\right)^{l_{1}}\left(x_{2} x_{2}\right)^{l_{2}} \cdots\left(x_{\ell} x_{\ell}\right)^{l_{\ell}}
$$

satisfies the first property. The second and third properties follow from the fact that $\left(x_{i} x_{i}\right)^{\left(p^{m_{i}}\right)}$ acts as the nonzero scalar $b_{i}^{2}$ on $\mathscr{P}$. If $\lambda_{\mathrm{n}}=\lambda_{\mathrm{n}}$, then by (3.4) each $l_{i}$ is divisible by $p$ and $p$-powers of root vectors are central. 
For $\mathfrak{g}(A)$, we have that $x_{i} \partial_{t}$ is a root vector which acts injectively for any $i=1,2, \ldots, \ell$ with $i \neq t$ because $\left(x_{i} \partial_{t}\right)^{\left(p^{\left.m_{i}+m_{t}\right)}\right.}$ acts as the nonzero scalar $b_{i}^{\left(p^{m_{t}}\right)} b_{t}^{\left(-p^{\left.m_{i}\right)}\right.} \tilde{a}_{t}^{\left(p^{m_{i}+m_{t}-1}\right)}$. For any $i=1,2, \ldots, \ell$, let $l_{i}$ be the unique integer between 0 and $p^{m_{i}}-1$ congruent to $\bar{n}_{i}-n_{i}$ modulo $p^{m_{i}}$. Assume that $m_{r}=\min \left(m_{1}, m_{2}, \ldots, m_{\ell}\right)$. Because $x^{\mathbf{n}}$ and $x^{\overline{\mathbf{n}}}$ have the same degree, $c:=\sum_{i=1}^{\ell}\left(\bar{n}_{i}-n_{i}\right)$ is divisible by $p^{m_{r}}$. Thus, there exists a nonzero scalar $s$ such that

$$
\sigma_{\mathrm{nin}}=1 / s\left(x_{1} \partial_{t}\right)^{l_{1}} \cdots\left(x_{r} \partial_{t}\right)^{l_{r}-c} \cdots\left(x_{\ell} \partial_{t}\right)^{l_{l}}
$$

satisfies the desired properties. If $r=t$, then there is no need to correct any exponent by subtracting $c$.

Using the operators $\sigma_{\text {nñ }}$ of the lemma, we see that every submodule of $\mathscr{P}$ $\left(\mathscr{P}_{j}\right)$ also has the full complement of $p^{\ell}\left(p^{\ell-1}\right)$ distinct weights and also has weight spaces of the same dimension. In particular, we have the following.

Theorem 3.6. The $\mathfrak{g}(C)$ representation $\mathscr{P}(\mathbf{1}, \mathbf{a}, \mathbf{b})$ is irreducible of degree 1 . If $a_{t} \notin k_{p}$ for some $t=1,2, \ldots, \ell$, then the $\mathfrak{g}(A)$ representation $\mathscr{P}_{j}(\mathbf{1}, \mathbf{a}, \mathbf{b})$ is irreducible of degree 1 for any $j=0,1, \ldots, p^{\hat{m}}-1$.

By Theorem 3.3, the irreducible representations $\mathscr{P}(\mathbf{1}, \mathbf{a}, \mathbf{b})$ and $\mathscr{P}_{j}(\mathbf{1}, \mathbf{a}, \mathbf{b})$ are nonrestricted. Since irreducible $\mathfrak{g}$ representations are frequently of finite degree with all possible $p^{\text {rank }(g)}$ weights, the previous theorem suggests that these newly constructed modules are of minimal possible dimension among all nonrestricted modules for these algebras. The study of this question and its relation to the Kac-Weisfeiler conjecture [WK] on the maximal $p$-power dividing the dimension of a modular representation are considered by Peters $[\mathrm{P}]$ in a related paper. In that work, the characters of these irreducible representations are analyzed and are shown to be nilpotent.

Under the stronger assumption that $a_{i} \notin k_{p}$ for all $i=1,2, \ldots, \ell$, Theorems 3.3 and 3.6 show that $\mathscr{P}(\mathbf{1}, \mathbf{a}, \mathbf{b})$ and $\mathscr{P}_{j}(\mathbf{1}, \mathbf{a}, \mathbf{b})$ are pointed torsion free representations of $\mathfrak{g}(C)$ and $\mathfrak{g}(A)$, respectively. In another related paper, Shi $[\mathrm{S}]$ shows that the only classical simple Lie algebras with such representations are those of type $A$ and $C$ (compare with the characteristic 0 work of Fernando [F]). Furthermore, for Lie algebras of these types, $\mathscr{P}(\mathbf{1}, \mathbf{a}, \mathbf{b})$ and $\mathscr{P}_{j}(\mathbf{1}, \mathbf{a}, \mathbf{b})$ exhaust all the possibilities.

The representations formed by truncation at higher levels do not remain irreducible and do not admit characters but are indecomposable of arbitrary high dimension.

Theorem 3.7. The $\mathfrak{g}(C)$ representation $\mathscr{P}(\mathbf{m}, \mathbf{a}, \mathbf{b})$ has a unique minimal submodule isomorphic to $\mathscr{P}(\mathbf{1}, \mathbf{a}, \mathbf{b})$ and the $\mathfrak{g}(A)$ representation $\mathscr{P}_{j}(\mathbf{m}, \mathbf{a}, \mathbf{b})$ has a unique minimal submodule isomorphic to $\mathscr{P}_{j}(\mathbf{1}, \mathbf{a}, \mathbf{b})$.

Proof. Let $V$ be any irreducible submodule of $\mathscr{P}$. Choose any weight $\lambda$ of $\mathscr{P}$, and let $\left\{x^{\mathbf{n}_{1}}, x^{\mathbf{n}_{2}}, \ldots, x^{\mathbf{n}_{r}}\right\}$ be the monomial basis of the $\lambda$ weight space of $\mathscr{P}$. As a consequence of Lemma 3.5, $\lambda$ is a weight of $V$. Let

$$
v=\sum_{i=1}^{r} \alpha_{i} x^{\mathbf{n}_{i}}
$$

for $\alpha_{i} \in k$ be a nonzero vector of $V$ in the $\lambda$ weight space. For $j \neq 1$, let $\sigma$ be the operator $\sigma_{\mathbf{n}_{j} \mathbf{n}_{1}}$ of Lemma 3.5. The central element $\sigma$ must act as 
some nonzero scalar $s_{\sigma}$ on the irreducible $V$. But $\sigma^{\left(p^{|m|}\right)}$ acts as some scalar $s$ on all of $\mathscr{P}$. This implies $s_{\sigma}$ is the unique $p^{|\mathrm{m}|}$ root of $s$ and as such is independent of the choice of irreducible $V$. Now

$$
s_{\sigma} v=\sigma(v)=\sum_{i=1}^{r} \alpha_{i} \sigma\left(x^{\mathbf{n}_{i}}\right) .
$$

Equating coefficients of the $x^{\mathbf{n}_{1}}$ terms yields $\alpha_{1} s_{\sigma}=\alpha_{j}$, proving that $\alpha_{j}$ is completely determined by $\alpha_{1}$ and is independent of the irreducible $V$. It follows that every irreducible module must contain the vector $v$, proving $\mathscr{P}$ has a unique minimal submodule. Furthermore, the mapping

$$
x^{\mathrm{n}} \longmapsto x^{\mathrm{n}}+\sum_{\substack{\boldsymbol{\nu} \equiv \mathbf{n} \\ \nu \neq \mathbf{n}}} s_{\sigma_{\nu}} x^{\nu}
$$

extends to an isomorphism from $\mathscr{P}(\mathbf{1}, \mathbf{a}, \mathbf{b})$ to the unique irreducible submodule of $\mathscr{P}$.

The proof for the $\mathfrak{g}(A)$ representation $\mathscr{P}_{j}$ is analogous.

\section{ACKNOWLEDGMENT}

The authors wish to thank Georgia Benkart for her insight into this subject and for facilitating this joint effort. Some of the results of this paper are contained in the dissertation of Karl Peters written under her guidance at the University of Wisconsin-Madison.

\section{REFERENCES}

[B] S. Berman, On the construction of simple Lie algebras, J. Algebra 27 (1973), 138-183.

[BFL] D. J. Britten, V. M. Futorny, and F. W. Lemire, Simple finite dimensional $C\left(A_{2}\right)$ modules (to appear).

[BL1] D. J. Britten and F. W. Lemire, The irreducible representations of $A_{n}$ with a 1-dimensional weight spaces, Trans. Amer. Math. Soc. 273 (1982), 509-540.

[BL2] _ A classification of simple Lie modules having a 1-dimensional weight space, Trans. Amer. Math. Soc. 299 (1987), 683-679.

[D] J. Dixmier, Enveloping algebras, North-Holland, Amsterdam, 1977.

[F] S. L. Fernando, Lie algebra modules with finite dimensional weight spaces. I, Trans. Amer. Math. Soc. 322 (1990), 757-781.

[FP1] Eric M. Friedlander and Brian J. Parshall, Modular representation theory of Lie algebras, Amer. J. Math. 110 (1988), 1055-1094.

[FP2] — Deformations of Lie algebra representations, Amer. J. Math. 112 (1990), 375-395.

[FP3] Induction, deformation, and specialization of Lie algebra representations, Math. Ann. 290 (1991), 473-489.

[KW] V. G. Kac and B. Weisfeiler, Cojoint action of a semisimple algebraic group and the center of the enveloping algebra in characteristic p, Indag. Math. 38 (1976), 135-151.

[P] Karl M. Peters, Characters of modular torsion free representations of classical Lie algebras, Comm. Algebra (to appear).

[S] Zhiyong Shi, Classification of pointed weak torsion free representations for classical Lie algebras, J. Algebra (to appear).

[SF] H. Strade and R. Farnsteiner, Modular Lie algebras and their representations, Marcel Dekker, New York, 1988. 
[WK] B. Weisfeiler and V. Kac, The irreducible representations of Lie p-algebras, Functional Anal. Appl. 5 (1971), 471-503.

[Z] H. Zassenhaus, The representations of Lie algebras of prime characteristic, Proc. Glasgow Math. Assoc. 2 (1954), 1-36.

Department of Mathematical Sciences, Loyola University Chicago, Chicago, Illinois 60626

E-mail address: kmpemath.luc.edu

Department of Mathematics, University of Western Ontario, London, Ontario, CANADA N6A 5B7

E-mail address: zshi@uwovax.uwo.ca 